similarly studied. Here, however, addition of inhibitor leads to no significant shifts, and it therefore seems likely that in this enzyme histidines are not directly involved in the active site. The $p \mathrm{~K}$ values of the four residues have been measured, and one-the least readily protonated-shows evidence of interaction with calcium ions.

A minute study of the NMR spectrum of lysozyme has been described by Sternlicht and Wilson (Biochemistry, 6, 2881; 1967). This represents a beginning to the formidable task of allocating all the observed resonances: from the crystallographic co-ordinates it has been possible to calculate interactions (for example, ring-current shifts of methyl protons close to aromatic rings), which has made possible a near-certain identification of several signals from side chains and backbone. The changes occurring on thermal unfolding have been described.

A review, including much new work, of results obtained with the celebrated new $220 \mathrm{Mc}$ Varian instrument also requires mention (Ferguson and Phillips, Science, 157, 257; 1967). This shows yet again, and in spectacular fashion, the resolution of the four histidine residues of ribonuclease, and the changes observed when ribonuclease and lysozyme are denatured. It will be noted that all the other work which has been mentioned was performed with 60 or 100 Mc instruments. Wider applications in enzymology may be expected.

\section{More about Chain Initiation}

\section{from our Cell Biology Correspondent}

GIVEN the great variety of proteins in ribosomes, it has always been a puzzle why ribosomes consist of two subunits; there seems to be no a priori reason why a single particle could not fulfil all the ribosomal functions. To explain the existence of subunits it has been suggested that during protein synthesis there is a cycle involving their separation and also that there is some division of the ribosomal functions between them. Several recent experiments, most of which are reported in the last two numbers of Proc. US Nat. Acad. Sci., confirm both these suggestions.

Nomura and his collaborators (Proc. US Nat. Acad. $S_{c i}$., 58, 946 and 1487; 1967), Eisenstadt and Brawerman (ibid., 1560) and Hille et al. (ibid., 1652) have all reported on the specific role of the $30 S$ ribosomal subunit in the initiation of polypeptide chain synthesis in $E$. coli. The following general scheme emerges from their data-which are in gratifying agreement-and from previous results. First, initiation factors, which stimulate formyl methionyl $t \mathrm{RNA}_{\mathrm{F}}$ binding to ribosomes programmed with the initiating codons AUG or GUG, bind to the $30 S$ subunit. Then the $30 S$ subunit binds $m$ RNA and F-met $t$ RNA to form an initiation complex, an obligatory intermediate of initiation. It is now generally agreed (Ohta, Sarkar and Thach, ibid., 1638) that this binding of F-met $t$ RNAF is GTP dependent although GTP may not be split at the binding stage but during the formation of the first peptide band. And according to Economou and Nakamoto (ibid., 1033) the methionyl residue is formulated before it binds to a ribosome, but this is still a disputed point. Once the initiation complex has formed a $50 S$ subunit, it couples with it to produce a $70 \mathrm{~S}$ ribosome attached to $m \mathrm{RNA}$ and with the chain initiator in place. Only when this has happened can other amino-acyl $t$ RNA bind and the first peptide bond be formed. The requirement of the $50 S$ subunit for peptide bond formation is in agreement with Munro's results ( $J$. Mol. Biol., 26, 147; 1967) which suggest that the enzyme necessary for this, peptidyl transferase, resides in the $50 \mathrm{~S}$ subunit. Once polypeptide synthesis has been initiated. Fisenstadt and Brawerman envisage the initiation factors leaving the $70 S$ ribosome and binding to another free $30 S$ subunit. This would explain why initiation factors must be added to get initiation in vitro with $70 S$ ribosomes while the $30 S$ subunit forms an initiation complex without added factors and can then couple with a $50 \mathrm{~S}$ subunit. It also explains why initiation factors are not found free in the soluble extracts of $E$. coli.

At chain termination and release of the completed protein the $70 \mathrm{~S}$ ribosome dissociates into $30 \mathrm{~S}$ and $50 \mathrm{~S}$ subunits and if the $30 S$ subunit picks up more initiation factor the cycle can be repeated. Mangiarotti and Schlessinger's (1966) discovery that the ribosomal population of $E$. coli consists of free $30 \mathrm{~S}$ and $50 \mathrm{~S}$ subunits and polysomes without a pool of free and stable $70 \mathrm{~S}$ ribosomes clearly provides evidence for this. Moreover, Schlessinger et al. (ibid., 1782) now report that free $30 S$ and $50 S$ ribosomal subunits from $E$. coli do not spontaneously associate to form $70 \mathrm{~S}$ ribosomes but will associate in the presence of $m \mathrm{RNA}$, $t$ RNA, $\mathrm{K}^{+}$and $\mathrm{Mg}^{++1}$ ions. The $70 S$ ribosomes seem to be stabilized by peptidyl $t \mathrm{RNA}$, for when peptide chain release is effected with puromycin they dissociate into $30 S$ and $50 S$ subunits.

So far, of course, all the experimental evidence for this scheme comes from work with $E$. coli, but it is unlikely that the process of protein synthesis should differ fundamentally in eucells. Indeed, there have been several reports-the latest by Sells and Takahashi (Biochim. Biophys. Acta, 134, 69; 1967)-that in nucleated cells $m$ RNA associates with the small ribosomal subunit in the nucleus and then migrates to the cytoplasm; it would be no surprise if these complexes prove to bo initiation complexes involving newly synthesized $m$ RNA and small ribosomal subunits.

\section{The Genetic Code in vivo}

\section{by our Molecular Genetics Correspondent}

THE detailed relationship between amino-acids and their trinucleotide codons has been established for Escherichia coli over the past six years. Of the sixtyfour codons, three are nonsense (UAG, UAA, and UGA) and the remainder code for the twenty natural aminoacids. The evidence for constructing this dictionary of codons and amino-acids has come essentially from two different types of biochemical experiments, both carried out in the test-tube. The first of these is the triplet binding technique in which a single trinucleotide is used to select and bind the correct transfer-RNA molecule carrying its amino-acid to the ribosome. This experimental approach, developed in Nirenberg's laboratory, is limited because of the rather small effects observed. The second experimental set-up, developed in Khorana's laboratory, utilized defined messenger RNAs with simple repeating sequences to direct the synthesis of polypeptides whose sequence was then determined. This, then, has provided us with the genetic code of $E$. coli in vitro.

It is far from easy to establish that this is exactly 